\title{
El papel de la movilidad estudiantil en el desarrollo de la competencia comunicativa intercultural: un estudio de caso
}

\section{The role of student mobility in the development of intercultural communicative competence: \\ a case study}

\author{
Marjana Šifrar Kalan \\ Univerza v Ljubljani (Eslovenia) \\ marjana.sifrar-kalan@ff.uni-lj.si
}

\begin{abstract}
The objective of this case study is to investigate the role of student mobility in the development of intercultural communicative competence in university students. In order to indicate the changes in the participants, especially about their attitudes towards Spanish culture, Spaniards and their stereotypes, an interpretative research with an ethnographic approach has been carried out, namely, we have interviewed several students of Hispanic Philology from the University of Ljubljana (level C1 of Spanish), who had spent at least one semester with the Erasmus scholarship studying in Spain. The article does not pretend to achieve a statistical generalization due to its limited sample, however it can be useful as a preliminary study.
\end{abstract}

Keywords: intercultural communicative competence, intercultural encounter, attitudes and stereotypes towards Spanish culture, student mobility

\section{INTRODUCCIÓN}

Teniendo en cuenta los esfuerzos que el Consejo de Europa y los países miembros desarrollan para que los intercambios estudiantiles universitarios contribuyan de manera eficaz al diálogo intercultural, al conocimiento y al respeto mutuo, esta 
investigación pretende analizar el desarrollo de la competencia comunicativa intercultural (CCI) de los estudiantes universitarios eslovenos que participan en el programa de movilidad Erasmus con una estancia en España de, al menos, un cuatrimestre de duración. Para ello, se ha llevado a cabo una investigación cualitativa de carácter interpretativo con un enfoque etnográfico, en la que se expone el cambio de actitudes que experimentan los participantes hacia la cultura española, los españoles y sus estereotipos tras su estancia en España. Los resultados obtenidos en esta investigación se han comparado con un estudio cuantitativo previo (Šifrar Kalan, 2020) en el que se confirma que la estancia Erasmus no influye significativamente en los estereotipos, así como que la imagen que tienen los universitarios eslovenos de un nivel avanzado de ELE sobre España, su cultura y lengua sigue siendo todavía estereotipada.

La primera parte del trabajo está dedicada a presentar sucintamente el concepto de la CCI en el contexto europeo de enseñanza de lengua extranjera, para lo cual partimos de una concepción amplia de interculturalidad: la posibilidad de diálogo entre culturas. A continuación, presentamos diversos estudios acerca del impacto de la movilidad estudiantil en esta competencia. En la parte empírica describimos la metodología cualitativa empleada en el estudio de caso y analizamos las entrevistas de los estudiantes eslovenos de Filología Hispánica después de su estancia Erasmus en España, de donde se deduce el desarrollo de la CCI. Puesto que se trata de un estudio de caso, no se pretende conseguir una generalización estadística debido al carácter limitado de la muestra; sin embargo, puede servir como estudio preliminar para pruebas más sistemáticas posteriores.

\section{LA COMPETENCIA COMUNICATIVA INTERCULTURAL}

El Consejo de Europa reconoce el desarrollo de la CCI como uno de los elementos clave en la sociedad democrática que respeta los derechos humanos y el diálogo intercultural y prepara a los jóvenes ciudadanos para convivir en un mundo lleno de diversidad. Con la promoción de proyectos y publicaciones sobre la CCI, se desea combatir el nacionalismo, el racismo, el discurso hostil, la discriminación, los prejuicios y los estereotipos (Huber \& Reynolds, 2014, pp. 7-9). Además, como señala Spychała (2014, p. 98), "en el mundo actual si no tenemos en cuenta las herramientas de la comunicación intercultural podemos perjudicar cada acto comunicativo", lo que, obviamente, puede propiciar conflictos, así como el aumento de la intolerancia.

Con el proceso de globalización del mundo, el concepto de la CCI ha adquirido más importancia. Aunque el MCER (2001/2002) ${ }^{1}$ no define de manera explícita la competencia intercultural (CI), subraya el desarrollo de la personalidad e identidad dentro de los ámbitos de diferentes lenguas y culturas $(2002$, p. 1) e introduce las

${ }^{1} 2001$ se refiere a la edición primera en inglés y francés, y 2002 se refiere a la versión española. 
competencias plurilingüe y pluricultural, con lo cual propone el abandono de la dicotomía entre la cultura propia y la cultura meta. La publicación complementaria y actualizada del MCER, el Companion Volume (2020), cuyo objetivo principal es "to promote plurilingual and intercultural education" (2020,p. 13), aporta una visión más detallada de la competencia plurilingüe y pluricultural, ya que en sus descriptores se aprecia un cierto grado de desarrollo de la CCI. De hecho, la integración de lo intercultural se refleja a través del empleo de numerosas expresiones, como intercultural encounters, understanding, exchange, mediator o competence.

Iglesias Casal \& Ramos Méndez (2021, p. 5) advierten que se producen confusiones y solapamientos terminológicos entre la CI y la CCI, pues mientras "algunos investigadores consideran la primera como parte integral de una competencia comunicativa intercultural más amplia (Byram, 1997), otros incluyen la CI como un subcomponente de la CC (Balboni, 2006) y, finalmente, otros las describen como competencias independientes (Deardorff, 2006)". Las autoras ofrecen una descripción perspicaz de la naturaleza y las dimensiones de estas competencias desde el punto de vista de los antecedentes, del estado actual de la cuestión y de las perspectivas de investigaciones futuras. Lo que estos términos intentan explicar es la capacidad de ir más allá de la propia cultura, de reconfigurar la visión original del mundo para poder comunicarse de manera competente con individuos de orígenes lingüística y culturalmente diversos. En un sentido más amplio, siguiendo a Fantini (2007, p. 9), la CCI se puede definir como "un complejo de habilidades necesarias para desempeñarse de manera efectiva y apropiada al interactuar con otros que son lingüística y culturalmente diferentes a uno mismo"2. Sin embargo, cambiar de perspectiva cultural se hace más difícil cuando los aprendices de la lengua extranjera ya han adquirido la de su lengua y cultura maternas; de ahí el poder de la estancia intercultural para brindar una oportunidad de "mirar el mundo con otros ojos" (Fantini, 2007, p. 8).

La definición más detallada de la CCI es la publicada por el Consejo de Europa (Huber \& Reynolds, 2014), que sostiene lo siguiente:

Intercultural competence is therefore a combination of attitudes, knowledge, understanding and skills applied through action which enables one, either singly or together with others, to:

- understand and respect people who are perceived to have different cultural affiliations from one self;

- respond appropriately, effectively and respectfully when interacting and communicating with

such people;

- establish positive and constructive relationships with such people;

- understand oneself and one's own multiple cultural affiliations through encounters with cultural difference (Huber \& Reynolds, 2014, pp. 16-17).

\footnotetext{
${ }^{2}$ La traducción es nuestra.
} 
Si bien las actitudes, el conocimiento, la comprensión y las habilidades son componentes necesarios de la competencia intercultural, poseer estos componentes por sí solo no es suficiente para que se le atribuya a un individuo la CCI: también es necesario que estos componentes se implementen y se pongan en práctica mediante la acción durante los encuentros interculturales. En nuestro caso, pretendemos averiguar cómo los estudiantes universitarios eslovenos pusieron en práctica sus actitudes, conocimientos y habilidades durante su estancia Erasmus en España.

\section{EL PAPEL DE LA MOVILIDAD ESTUDIANTIL}

Huber \& Reynolds (2014, pp. 27-28) señalan que la CCI se puede desarrollar a través de tres tipos de educación: (1) informal (familia, amigos, compañeros, etc.), (2) no formal (educación programada fuera del contexto educativo formal como ONG, trabajo juvenil, etc.), (3) formal (todas las instituciones de educación formal vertical). En nuestro caso, el intercambio Erasmus pertenece, sobre todo, a la educación formal y al mismo tiempo a la informal, con sus compañeros de curso y de piso, por ejemplo. Según la Guía del programa de Erasmus + (2017, pp. 9-28), los objetivos del programa de intercambio son, entre otros, promover el aprendizaje de las lenguas y la diversidad lingüística, así como reflexionar sobre culturas extranjeras y la suya propia para, de este modo, desarrollar la sensibilización intercultural.

Kinginger (2013, pp. 4-8) señala que muchos estudios sobre el aprendizaje de la LE en el extranjero muestran que estudiar en el extranjero tiene la capacidad potencial de aprender el idioma, por un lado, y, por otro lado, brinda la gran oportunidad de contactos sociales auténticos que la clase nunca puede ofrecer. Añade que otra conclusión de los estudios previos sobre el tema es que se subrayan las diferencias individuales significativas. Estas diferencias individuales se corroboran con el estudio de 96 estudiantes alemanes de Erasmus (Hessel, 2019), en el que se destaca la importancia de las autoconcepciones de la LE de los estudiantes en interacciones sociales durante su estancia en el extranjero. Por su parte, el estudio de Hernández (2010) con un grupo de estudiantes extranjeros que estuvieron un semestre en España sugiere que las interacciones interculturales durante los estudios en el extranjero pueden mejorar la motivación en el aprendizaje de la LE y, consecuentemente, promover el aprendizaje intercultural. Asimismo, la investigación de la Universidad de Oviedo sobre las representaciones culturales de estudiantes extranjeros en España subraya el carácter evolutivo que la estancia ha ejercido en sus representaciones culturales (Iglesias Casal, 2015, p. 76). A pesar de los muchos beneficios que aporta al estudiante la inmersión en una comunidad de lengua extranjera, tenemos que darnos cuenta también de que la mejora no ocurre en todos los aspectos; de hecho, diferentes estudios recopilados en Šifrar Kalan (2020) y este mismo estudio señalan que los estereotipos pueden permanecer. 


\section{METODOLOGÍA}

La investigación está basada en el enfoque cualitativo del estudio de caso, puesto que el objetivo principal de este tipo de exploración es "encontrar individuos que pueden proveer observaciones ricas y variadas sobre el fenómeno bajo investigación para maximizar lo que se puede aprender" (Dörnyei, 2007, p. 126) ${ }^{3}$. Por este motivo hemos trabajado con una muestra pequeña $(\mathrm{N}=5)$, que nos ha permitido analizar los datos con mayor grado de detalle. Para la selección de los informantes que han participado en la investigación se ha llevado a cabo un muestreo no probabilístico de conveniencia, ya que se invitó a todos los estudiantes del último curso de grado y del programa de máster de la Facultad de Filosofía y Letras de la Universidad de Ljubljana que realizaron una estancia Erasmus en España entre los años 2017 y $2019^{4}$.

Como se puede observar en la tabla 1 , se trata de un grupo bastante homogéneo, que representa al estudiante típico de los cursos avanzados de Filología Hispánica de la Universidad de Ljubljana: todas son mujeres con edades comprendidas entre los 23 y los 26 años y con un nivel $\mathrm{C} 1$ de español, excepto una informante que tiene nivel B1. El nivel está determinado por el examen que tienen que realizar antes de su estancia Erasmus, que también repiten cuando vuelven y en el que todas las participantes presentan un nivel $\mathrm{C} 1$ o $\mathrm{C} 2$. Por tanto, cuatro de ellas progresan en el nivel, menos una, que se queda con un $\mathrm{C} 1$. Por último, dado que muchas preguntas se refieren a su inclusión en la nueva sociedad, a los nuevos contactos sociales, creemos que es importante tener en cuenta también su estimación de su nivel de sociabilidad (de 1 a 5).

Tabla 1. Descripción de la muestra

\begin{tabular}{|c|c|c|c|c|c|}
\hline MUESTRA & A & B & $\mathrm{C}$ & $\mathrm{D}$ & E \\
\hline Edad & 26 & 23 & 23 & 25 & 25 \\
\hline Conocimiento de ELE & 6 & 4 & 6 & 5 & 5 \\
\hline Curso de grado/máster & Fin de máster & Fin de grado & $\begin{array}{l}3 .^{\text {er }} \text { curso de } \\
\text { grado }\end{array}$ & $\begin{array}{l}\text { 1. }{ }^{\text {er }} \text { curso de } \\
\text { máster }\end{array}$ & $\begin{array}{l}\text { 1. }{ }^{\text {er }} \text { curso de } \\
\text { máster }\end{array}$ \\
\hline $\begin{array}{c}\text { Años de aprendizaje } \\
\text { de ELE }\end{array}$ & 13 & 8 & 8 & 12 & 12 \\
\hline $\begin{array}{c}\text { Nivel antes/después de } \\
\text { Erasmus }\end{array}$ & $\mathrm{C} 1 / \mathrm{C} 2$ & $\mathrm{~B} 1 / \mathrm{C} 1$ & $\mathrm{C} 1 / \mathrm{C} 2$ & $\mathrm{C} 1 / \mathrm{C} 1$ & $\mathrm{C} 1 / \mathrm{C} 2$ \\
\hline $\begin{array}{l}\text { Meses de estancia } \\
\text { Erasmus }\end{array}$ & 5 & 5 & 9 & 10 & 5 \\
\hline Lugar de Erasmus & Valencia & Logroño & Sevilla & Granada & Murcia \\
\hline Curso escolar & 2018-2019 & $2017-2018$ & 2018-2019 & 2017-2018 & 2017-2018 \\
\hline $\begin{array}{l}\text { Grado de sociabilidad } \\
\qquad(1-5)\end{array}$ & 5 & 4 & 3 & 3 & 4 \\
\hline
\end{tabular}


El instrumento utilizado ha sido la entrevista semiestructurada que, según Dörnyei (2007, p. 136), significa que "hay una serie de preguntas orientadoras preparadas de antemano [...] [pero al mismo tiempo] el formato es abierto y se alienta al entrevistado a que ahonde más en las cuestiones planteadas de modo exploratorio"s. En todas las entrevistas llevadas a cabo fueron formuladas las mismas 32 preguntas que habían sido preparadas y pilotadas con antelación. No obstante, durante cada entrevista individual también fueron añadidas algunas preguntas adicionales con el objeto de precisar o añadir alguna aclaración. Las entrevistas se realizaron en la primavera de 2020 vía Skype en esloveno, la lengua materna de las estudiantes. El promedio de duración de las entrevistas fue de 60 minutos. Las conversaciones fueron grabadas y transcritas. Para el diseño del cuestionario, nos basamos parcialmente en Fantini (2007), en Huber $\&$ Reynolds (2014) y en un estudio parecido al nuestro sobre la influencia de la movilidad en la CCI en los estudiantes serbios de español (Mastilo, 2011).

\section{ANÁLISIS DE LAS ENTREVISTAS}

Las respuestas, que siguen los componentes de la CCI según Huber \& Reynolds (2014, pp. 16-21), se presentan por sus pseudónimos (A-E) en cuatro grupos de preguntas y se confrontan con investigaciones similares.

Las respuestas a las primeras siete preguntas atañen, sobre todo, a sus primeros contactos y experiencias, sus actitudes y conocimientos sobre la lengua y cultura españolas antes de su estancia Erasmus. Entre sus actitudes, tratamos de evaluar si aprecian la diversidad cultural, si muestran curiosidad y disponibilidad de aprender de y sobre la gente de otras culturas y si buscan oportunidades para encuentros interculturales. Podemos observar que todas las estudiantes presentan una actitud muy positiva hacia la lengua y cultura españolas, ya que todas (excepto una) muestran mucha curiosidad por aprender otra lengua y cultura. Creemos que una de las razones de la actitud positiva hacia lo diferente se debe a lo que Iglesias Casal $(2015$, p. 63) llama razón geopolítica, puesto que Eslovenia es un país pequeño con solo dos millones de habitantes y hablantes de esloveno, donde prevalecen una conciencia y necesidad muy altas de abrirse al mundo.

Todas las informantes tienen en común la búsqueda de contactos con lo español, o en forma de viajes, o a través de lectura, música o redes sociales. De hecho, cuatro de ellas entraron por primera vez en contacto con el español a través de la televisión, mientras que solo una lo hizo en su entorno familiar. El factor decisivo para elegir el instituto de enseñanza secundaria fue para todas poder aprender español como L2 o L3. Su actitud positiva se verbaliza con frases como "todo lo conectado con el español se convirtió en una pasión", "no me enamoré solo de la lengua española

\footnotetext{
${ }^{5}$ La traducción es nuestra.
} 
sino también de su arquitectura, el ritmo dinámico de la calle". La conciencia de la diversidad cultural española está presente en cuatro de las cinco informantes, debido a que hicieron viajes cortos a España antes de su estancia Erasmus, mientras que una informante admite que tenía una imagen ingenua de "España soleada". Igualmente, Iglesias Casal (2015, p. 74), según los resultados de una investigación en la Universidad de Oviedo con 168 estudiantes extranjeros, subraya el papel imprescindible de los viajes, que les permitió superar la visión monolítica de España. Las respuestas de nuestras informantes a la pregunta "¿por qué decidieron participar en el programa Erasmus?" coincide mucho con los hallazgos de Lesjak et al. (2015, p. 854), quienes consideran que es favorable para su desarrollo personal y profesional, y mencionan motivos más concretos: experimentar algo nuevo, crecer personalmente, aprender sobre diferentes culturas, conocer gente nueva, entre otros.

Las respuestas a las preguntas de la 8 a la 12 describen la adaptación de las estudiantes a una vida nueva durante su estancia Erasmus en España, su habilidad de comprender y enfrentarse con lo nuevo e inseguro, y si para esto tenían suficiente conocimiento previo de la cultura española.

La informante A elogia la acogida que recibieron los estudiantes Erasmus en la Universidad de Valencia, ya que obtuvieron muy buen asesoramiento para adaptarse a su vida allí. También considera que le resultó muy útil su experiencia previa de aupair con una familia andaluza y lo que aprendió sobre la cultura española antes en las clases en Eslovenia, razón por la que estaba preparada para los cambios.

Asimismo, la informante B también relata una acogida muy positiva en la Universidad de la Rioja. Explica que la preparación previa para la estancia en España y su deseo y determinación de vivir una experiencia nueva la ayudaron a superar el primer mes, que fue el más difícil.

En cambio, la informante $\mathrm{C}$ considera que no recibió una acogida tan positiva por parte la Universidad de Sevilla; además, le costó adaptarse a las clases plenarias, donde no se esperaba la participación activa de los estudiantes, y echó en falta el trabajo continuo para las clases, una experiencia totalmente contraria a las demás informantes.

Por su parte, la informante D afirma que le resultó difícil adaptarse, ya que no conocía a nadie en Granada. Al Igual que a la informante C, le costó acostumbrarse a que las tiendas estuvieran cerradas por la tarde (lo que no ocurre en Eslovenia), al horario de la comida y a conocer a tanta gente nueva.

Por último, la informante $\mathrm{E}$ se adaptó bastante rápido en Murcia y muy pronto entabló nuevas amistades, pues, como esperaba, la gente era muy abierta y amable.

De lo anterior podemos concluir que la acogida que las universidades ofrecen a los estudiantes Erasmus es de suma importancia. Todas las participantes coinciden en que recibieron la formación adecuada sobre la cultura española durante su carrera de Filología Hispánica, lo que es un dato importante y deseado para los profesores de ese programa. Este grado de satisfacción por parte de las estudiantes eslovenas coincide con un estudio similar (Mastilo, 2011) realizado con universitarios serbios. Nos 
referimos en este punto a una conclusión bastante generalizada, pero confirmada en la investigación que describe Iglesias Casal (2015, p. 64): "La formación más o menos académica es, naturalmente, fuente importante de las representaciones culturales".

Las respuestas siguientes (13-26) pretenden medir el contacto con los españoles y el español dentro y fuera del ámbito universitario y su percepción de sentirse acogidas. Más concretamente se trata de analizar si el hecho de ser extranjero y de nacionalidad eslovena influía en la reacción de los españoles o si tal vez tenían más contacto con otros extranjeros. En este sentido, se investigó si las relaciones sociales y los potenciales conflictos con los españoles cambiaron su opinión y estereotipos sobre ellos.

Todas las informantes coinciden en el hecho de que se sentían acogidas en el nuevo entorno a pesar de que los españoles no sabían nada de Eslovenia e incluso los profesores universitarios lo confundían con Eslovaquia o Estonia. Una de las informantes cuestiona el papel de la Unión Europea como una entidad social y política si sus miembros ni siquiera se conocen entre ellos. Tres de las informantes se sintieron decepcionadas al comprobar que los españoles no mostraban curiosidad por Eslovenia ni por la cultura eslovena. Un ejemplo de esto lo proporciona la informante $\mathrm{C}$, que no encontró en Sevilla el carácter abierto y amable que ella esperaba, ya que tenía la impresión de que los españoles estaban hartos de turistas y extranjeros, incluso en la universidad, donde había muchos estudiantes Erasmus.

En cuanto al contacto social entre los estudiantes Erasmus y los estudiantes españoles, todas las informantes sugieren que sería mucho mejor si los profesores lo fomentaran en sus clases. No cabe duda de que las informantes son conscientes de que la interculturalidad no es un proceso de sentido único, que "consiste simplemente en reconocer, descubrir o tolerar al otro y sus diferencias; se trata del intercambio, donde sean los mismos individuos quienes compartan conocimientos y experiencias" (Alfonzo de Tovar, 2020, p. 3). Según esta autora, el nivel educativo de los estudiantes (en nuestro caso se trata de estudiantes del último curso del grado y del máster) incide especialmente en la actitud y la conciencia (2020, p. 14).

Por lo que respecta a los casos de conflictos comunicativos, las informantes supieron mediar en estas situaciones. Todas subrayan que los conflictos comunicativos personales que surgieron durante su estancia no les han hecho cambiar la opinión de que los españoles son amables, alegres y sonrientes. En general, no cambiaron su percepción sobre cómo son los españoles, sino que la fortalecieron.

Con relación a los estereotipos de la cultura española, el hecho de que los españoles sean considerados personas muy habladoras lo justifica una de las informantes basándose en que no les gusta el silencio, y otra indica que les encanta "hablar por hablar, sí" pero no se puede tener una conversación abierta con argumentos a favor y en contra sobre un tema político (como la independencia de Cataluña) con los andaluces. Por otro lado, todas las informantes afirman haber comprendido el concepto de "la siesta" en un sentido más pleno como consecuencia de haber vivido el horario español y, aunque reconocen que sigue siendo un estereotipo, lo perciben de manera 
más positiva. También coinciden en la ruptura del estereotipo de que los españoles no trabajan mucho (también por la siesta); al contrario, solo tienen un horario diferente y dividen el trabajo durante todo el día. Sin embargo, la informante de Granada menciona los problemas burocráticos que le recordaban el artículo "Vuelva usted mañana" de Mariana José de Larra.

Una última cuestión que se trató en este bloque de preguntas fue el grado de sociabilidad de las participantes. Todas ellas, excepto una, participaron en las actividades de varias asociaciones externas a la universidad: un grupo de flamenco, un grupo de música, un grupo de LGBT y CSOA (Centro Social Ocupado Autogestionado). Esto les permitió establecer amistad tanto con estudiantes españoles como con otros estudiantes Erasmus. La informante con la autoevaluación más baja de sociabilidad (tabla 1), que además se mostró la más introvertida durante la entrevista, es la que menos contactos sociales tuvo, mientras que las demás tuvieron la oportunidad de desarrollar la CCI dentro del ámbito informal, no formal y formal, según Huber \& Reynolds (2014, pp. 27-28).

Las últimas preguntas, de la 27 a la 32, requerían una reflexión explícita sobre el desarrollo de su competencia intercultural: si al volver de España a su país, Eslovenia, notaban diferencia en su apertura, tolerancia y empatía hacia la gente y las culturas diferentes. Podemos concluir que la estancia en España excedió sus expectativas, ya que todas querían volver pronto. Aunque la estancia ha influido mucho en su desarrollo personal y académico, destacan la dimensión personal. Al ser ellas "diferentes" en España, ahora tienen más empatía hacia lo diferente. Subrayan que tienen una perspectiva más abierta hacia el mundo, y que los españoles les han "contagiado" su actitud positiva y alegre. "Cuantas más culturas conoces, más sensible y abierto eres para todo lo diferente", concluyó una de las estudiantes entrevistadas. Ante lo expuesto, queda de manifiesto "que en los programas de movilidad de larga duración como Erasmus+ se desarrollan, con más rapidez, la actitud y la conciencia intercultural" (Alfonzo de Tovar, 2020, p. 21).

\section{CONCLUSIONES}

Puesto que la muestra con la que se ha trabajado es no probabilística (compuesta por cinco informantes), no se pueden generalizar los resultados obtenidos y, por tanto, resulta imposible extrapolarlos a otras poblaciones; sin embargo, consideramos que el análisis resulta útil como estudio preliminar para futuras investigaciones más sistemáticas. Asimismo, este trabajo también puede utilizarse como elemento de comparación por parte de estudios contrastivos, que trabajen con muestras formadas por estudiantes de español como lengua extranjera procedentes de otros países. Además, los resultados obtenidos en la presente investigación son valiosos para los docentes involucrados en los programas de Filología Hispánica de la Universidad de Ljubljana, 
pues les permiten evaluar la idoneidad de la formación intercultural que ofrecen a sus estudiantes.

En cuanto a las limitaciones de este tipo de investigaciones cualitativas, estamos de acuerdo con Kinginger (2013, p. 5) en que los resultados positivos de una estancia académica en el extranjero pueden variar mucho, es decir, son muy subjetivos, y dependen de muchos factores. En el caso de nuestra muestra, muy pequeña y homogénea, podemos concluir que las estudiantes con un alto nivel de español (C1) y con una actitud previa positiva hacia otras culturas supieron mediar en los posibles conflictos comunicativos durante su estancia Erasmus en España y no dejaron que estos influyeran en su actitud positiva hacia la gente y cultura españolas. En las entrevistas relatan que han crecido personal y académicamente, y que después de esta estancia se sienten más abiertas y sensibles a lo diferente. Valoran esta experiencia como algo muy positivo en su desarrollo personal y como un cambio en la concepción de sí mismas, lo que confirma la evolución de la CCI. En este sentido, según Iglesias Casal (2015, p. 52), "el preguntarse por la identidad propia en relación a los otros forma parte integral del enfoque intercultural". Algunos estereotipos, como el de la siesta y los españoles amables y alegres, se han reforzado o confirmado, pero los entienden mejor dentro de su contexto. Por otro lado, todas mencionan que han roto con el estereotipo de los españoles como vagos y no trabajadores, al entender que se trata de un horario $\mathrm{y}$ una rutina diaria diferente.

Con este trabajo queríamos reflexionar sobre la importancia de la movilidad estudiantil en el desarrollo de la CCI de la que se benefician tanto los estudiantes a nivel académico y personal, como la sociedad en sentido más amplio. Por lo tanto, podemos concluir que su desarrollo compete tanto a las instituciones (como el Consejo de Europa y las universidades) como a los propios individuos, lo que resumen Huber \& Reynolds (2014, p. 53) de la siguiente manera: "It is also vital that people's intercultural competence is developed in order to enable them to understand, appreciate and respect each other across cultural differences, and to enable them to contribute actively to societies that benefit from diversity". En síntesis, cabe señalar que los estudiantes Erasmus no son los únicos que pueden sacar provecho de la estancia en otro país, sino también todas las personas con las que entren en relación. En este sentido, algunas estudiantes indican que echaban de menos la curiosidad y el interés por encuentros y diálogos interculturales por parte de los españoles. De hecho, es igualmente importante la acogida de las personas de otras culturas, ya que aprendemos o crecemos tanto cuando viajamos como cuando estamos acogiendo a quienes viajan. 


\section{BIBLIOGRAFÍA}

Alfonzo de Tovar, I.C. (2020). Desarrollo de la competencia intercultural en contextos plurilingües y pluriculturales: programa universitario de movilidad idiomática. Tonos Digital, 38 (I), 1-26.

Comisión Europea (2017). Erasmus+: Guía del programa. Recuperado de: https://ec.europa.eu/programmes/erasmus-plus/sites/erasmusplus2/files/2017-erasmus-plus-programme-guide-v2_es.pdf.

Consejo de Europa (2002). Marco común europeo de referencia para las lenguas: aprendizaje, enseñanza y evaluación. Madrid: Anaya, Instituto Cervantes, Ministerio de Educación.

Council of Europe (2020). Common European Framework of Reference for Languages: Learning, teaching, assessment. Companion Volume. Strasbourg: Council of Europe.

Dörnyei, Z. (2007). Research Methods in Applied Linguistics. Oxford: Oxford University Press.

Fantini, A. (2007). Exploring and Assessing Intercultural Competence. Research Report. Washington: Center for Social Develeopment. Recuperado de: https://openscholarship.wustl.edu/cgi/viewcontent. cgi?article $=1815 \&$ context $=$ csd_research.

Hernández, T.A. (2010). The relationship among motivation, interaction, and the development of second language oral proficiency in a study-abroad context. The Modern Language Journal, 94 (4), 600-617.

Hessel, G. (2019). Study Abroad: L2 Self-efficacy and Engagement in Intercultural Interactions. In A. Kostoulas (Ed.), Challenging Boundaries in Language Education, Second Language Learning and Teaching (pp. 199-210). Springer, Cham. DOI: 10.1007/978-3-030-17057-8_12.

Huber, J., Reynolds, C. (2014). Developing intercultural competence through education. Strasbourg: Council of Europe Publishing. Recuperado de: https://rm.coe.int/developing-intercultural-enfr/16808ce258.

Iglesias Casal, I. (2015). Entre tópicos y estereotipos: cómo se construyen y reconstruyen las representaciones culturales. Estudios Humanísticos. Filología, 37, 51-79.

Iglesias Casal, I., Ramos Méndez, C. (2021). Competencia comunicativa intercultural y enseñanza de español LE/L2: antecedentes, estado actual y perspectivas futuras. Journal of Spanish Language Teaching, 1-24. DOI: 10.1080/23247797.2020.1853367.

Kinginger, C. (Ed.) (2013). Social and cultural aspects of language learning in study abroad. Amsterdam: John Benjamins.

Lesjak, M., Juvan, E., Ineson, E., Yap, M., Axelsson, E. (2015). Erasmus student motivation: Why and where to go? Higher Education: The International Journal of Higher Education Research, 70 (5), 845-865.

Mastilo, M. (2011). Uticaj studentske mobilnosti na razvoj interkulturne komunikativne kompetencije kod studenata španskog kao stranog jezika (trabajo fin de máster). Universidad de Belgrado, Serbia.

Šifrar Kalan, M. (2020). Estereotipos culturales sobre España en estudiantes universitarios extranjeros: el caso de los Erasmus eslovenos. Ogigia. Revista electrónica de estudios hispánicos, 27, 213-234. DOI: 10.24197/ogigia.27.2020.213-234.

Spychała, M. (2014). La problemática de la comunicación intercultural: consideraciones didácticas para la enseñanza de las lenguas. Studia Romanica Posnaniensia, 41 (1), 87-100. DOI: 10.14746/ strop.2014.411.006. 